\title{
Kernos
}

Revue internationale et pluridisciplinaire de religion grecque antique

$27 \mid 2014$

Varia

\section{Renaud GAGNÉ, Ancestral Fault in Ancient Greece}

\section{Aurian Delli Pizzi}

\section{(2) OpenEdition \\ Journals}

Édition électronique

URL : http://journals.openedition.org/kernos/2235

DOI : $10.4000 /$ kernos. 2235

ISSN : 2034-7871

Éditeur

Centre international d'étude de la religion grecque antique

Édition imprimée

Date de publication : 1 novembre 2014

Pagination : 455-457

ISBN : 978-2-87562-055-2

ISSN : 0776-3824

\section{Référence électronique}

Aurian Delli Pizzi, «Renaud Gagné, Ancestral Fault in Ancient Greece », Kernos [En ligne], 27 | 2014, mis en ligne le 12 novembre 2014, consulté le 22 septembre 2020. URL : http://journals.openedition.org/ kernos/2235; DOI : https://doi.org/10.4000/kernos.2235

Ce document a été généré automatiquement le 22 septembre 2020.

Kernos 


\title{
Renaud GAGNÉ, Ancestral Fault in Ancient Greece
}

\author{
Aurian Delli Pizzi
}

\section{RÉFÉRENCE}

Renaud GAGNÉ, Ancestral Fault in Ancient Greece, Cambridge, Cambridge University Press, 2013. 1 vol. 16 × 23,5 cm, X+556 p. ISBN : 978-1-107-03980-3.

1 Derrière un titre assez sobre, la structure de l'ouvrage de R. Gagné (R.G.) permet d'entrevoir son originalité. Loin de se contenter d'une brève circonscription du concept de faute ancestrale dans l'introduction, l'A. consacre les deux premiers chapitres à la réception et à la réinterprétation de cette notion après l'Antiquité. Il cherche ainsi à montrer par quels filtres successifs a transité la notion avant d'arriver jusqu'à nous. Ce n'est qu'après un exposé assez érudit sur les nombreux auteurs impliqués dans la réception de la notion que l'A. revient à Homère, Hésiode et aux auteurs grecs postérieurs. L'arc chronologique de l'étude s'étend ainsi sur près de trois mille ans.

Cette structure répond à une question centrale : la notion-même de faute ancestrale est-elle une construction grecque ou moderne ? Cette question amène l'A. à distinguer deux facettes de cette même notion, selon qu'elle soit envisagée par les Grecs ou par nous : l'ancestral fault, du point de vue grec, et l'inherited guilt, du point de vue moderne. Cette distinction, quoique pertinente sur le plan méthodologique, est très subtile, au point que, au fil de l'ouvrage, l'A. utilise parfois l'une pour l'autre. Il souligne d'emblée les limites d'une approche cognitive qui consisterait à s'interroger sur ce que les Grecs "croyaient " à propos de la faute ancestrale et préfère se concentrer, à travers différents cas d'étude, sur les contextes d'énonciation dans lesquels apparaît la notion (p. 9).

Dans le premier chapitre, l'A. étudie le traitement de la notion de $\pi \rho o \gamma o v i k o ̀ v$

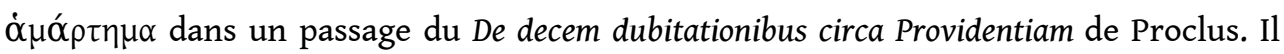
montre que Proclus s'est librement inspiré du traité De sera numinis vindicta de 
Plutarque, en infléchissant les idées pour les appliquer au contexte néoplatonicien. Il suggère que la réflexion autour de la notion de faute ancestrale ne s'est pas développée chez les Grecs avant la période hellénistique. Par la suite, selon lui, la réception de la notion par les auteurs chrétiens s'est structurée autour de deux types de réactions: l'appropriation ou le rejet. Ainsi, le rapport entre hellénisme et christianisme diffère selon les modes de traduction. La traduction d'Isaak Sebastokrator vise à s'approprier le texte (domestication translation), en établissant des liens avec l'Ancien Testament et

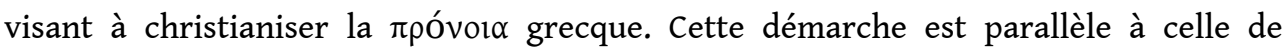
Michael Psellos, dans le contexte de la renaissance byzantine du $\mathrm{XI}^{\mathrm{e}} \mathrm{s}$. En revanche, la traduction de Guillaume de Moerbeke cherche davantage à mettre en lumière les différences du texte de Proclus (foreignisation translation), notamment en évitant de traduire en latin les termes grecs de Proclus.

4 Le deuxième chapitre étudie la réception de la notion de faute ancestrale aux $\mathrm{XVI}^{\mathrm{e}}$ et $\mathrm{XVII}^{\mathrm{e}} \mathrm{s}$., principalement à travers les éditions et commentaires concernant le traité de Plutarque. Ces travaux ont interprété le texte de Plutarque à l'aune du christianisme et ont contribué à façonner la notion d'inherited guilt, pendant moderne de l'ancestral fault. Hugo Grotius considère l'héritage de la culpabilité comme incompatible avec la liberté individuelle qu'il prône. Il distingue loi humaine et loi divine, et estime que la transmission de la culpabilité aux descendants n'est pas en adéquation avec la loi humaine. Jan Lomeier prend lui aussi Plutarque comme référence pour son traité sur les rites de purification. Bien qu'il distingue loi humaine et loi divine comme le fait Grotius, il considère que le péché originel ne peut être effacé que par la grâce divine, contrairement à la vision libérale de Grotius.

5 Après l'examen de ces deux auteurs, R.G. livre un aperçu de la recherche sur la notion d'inherited guilt après le $\mathrm{xvII}^{\mathrm{e}} \mathrm{s}$. La tendance est à considérer le péché originel comme de plus en plus marginalisé et comme une curiosité spécifique au monde grec. Cela a pour conséquence que l'on va chercher à déceler les origines et évolutions de cette croyance. L'A. s'intéresse surtout à la chaîne formée par les travaux successifs de G. Glotz, E. Dodds et H. Lloyd-Jones. Le déclin de la faute ancestrale s'inscrit parfaitement dans la

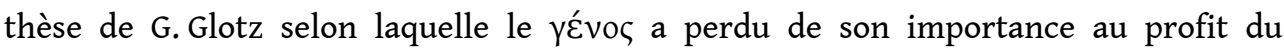
développement des institutions de la cité. Pour E. Dodds, la notion d'inherited guilt est caractéristique de la Grèce archaïque et entre dans la catégorie de l'irrationnel. Selon H. Lloyd-Jones, la notion est fondamentale dans la pensée grecque, même après la période archaïque.

6 Le troisième chapitre étudie l'ż $\xi \omega ́ \lambda \varepsilon \mid \alpha$, forme d'imprécation dirigée contre soi-même et contre son propre foyer, chez Homère et chez Hésiode. L'A. montre que, chez Hésiode, la culpabilité qui se diffuse aux générations suivantes est due au parjure. Le non respect d'un serment menace la continuité du foyer, tout en ayant un impact sur la cité. R.G. est bien conscient que la perspective dans laquelle s'insèrent ces serments, qui engagent les générations futures, est quelque peu différente du problème de la faute ancestrale à proprement parler. La faute ancestrale dans Les travaux et les jours se conçoit essentiellement en rapport avec la pratique rituelle du serment. L'A. fait une comparaison entre le parjure chez Hésiode et celui évoqué dans l'inscription de la fondation de Cyrène. Chez Homère, le grand serment des armées aux livres III et IV de l' Iliade, où le parjure des Troyens va les conduire à leur perte, montre également que le serment qui étend la responsabilité aux générations ultérieures est, plus qu'une simple 


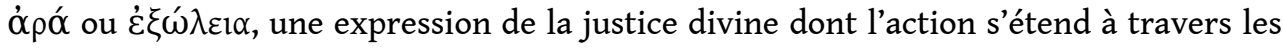
âges.

7 Le chapitre 4 aborde la faute ancestrale dans le contexte de la poésie récitée dans les banquets. $\mathrm{Au} \mathrm{VI}^{\mathrm{e}} \mathrm{s}$. av. J.-C., la faute ancestrale n'est plus confinée au registre des serments. Dans les poèmes d'Alcée, la culpabilité du tyran de Lesbos, Pittacos, est renforcée par l'attitude que son père et son grand-père ont eue avant lui, voire même par les fautes des Atrides, ses ancêtres, et son sacrilège est présenté comme susceptible d'avoir des répercussions sur ses descendants et sur sa cité. Les poèmes de Solon montrent tout le potentiel de l'implication des dieux dans la sphère humaine pour sévir contre une faute ancestrale: non seulement la notion de faute ancestrale s'étend au domaine de la cité entière, comme principe de gouvernance, mais des descendants peuvent être punis à la place de leurs parents. Enfin, malgré de fortes similitudes entre plusieurs passages de Solon et de Théognis, ce dernier se situe davantage dans la continuité d'Hésiode, avec une grande importance accordée au serment et à la notion d'intention, primordiale pour départager les responsabilités.

8 Après la poésie archaïque, la faute ancestrale n'est plus confinée au domaine du serment ou du banquet, mais s'étend dans différents domaines, comme le montre le chapitre 5. Elle sert comme élément narratif « to create an association between the past of the narrative and the present of the audience » (p. 306). L'épisode du serment de Glaukos, rapporté par Hérodote, mêle différentes problématiques pour illustrer l'impact d'un parjure sur les relations entre cités. Un autre cas est la colère du héros Talthybios, à Sparte, après que les Spartiates ont mis à mort des hérauts perses, colère qui a eu, selon Hérodote, des effets sur la guerre du Péloponnèse plusieurs décennies plus tard. L'épisode des Alcméonides est analysé à travers la représentation d'Hérodote, qui le présente comme un outil de manipulation par les autres cités à l'égard d'Athènes, et d'autres sources, comme la Constitution d'Athènes et la Vie de Solon de Plutarque, qui insistent davantage sur la purification. Enfin, Hérodote recourt à la faute ancestrale pour expliquer la chute de Crésus, malgré les avertissements d'Apollon et de Solon.

Dans les deux derniers chapitres, l'A. étudie le riche corpus des tragédies, en se concentrant sur deux familles : les Labdacides et les Atrides. Il procède à une étude au cas par cas, car il n'est pas possible de déceler une utilisation identique de la faute ancestrale dans toutes les attestations. La faute ancestrale est fort présente dans l'ensemble de la production tragique, contrairement à l'idée reçue qui voudrait qu'Eschyle, empreint d'archaïsme, en soit l'auteur le plus friand: ce point renforce l'affirmation de l'A. selon laquelle la faute ancestrale n'est pas un concept restreint à l'époque archaïque. Certes, la faute ancestrale est centrale dans l'Orestie d'Eschyle. C'est dans l'Agamemnon que les références au passé et au futur sont les plus riches. Clytemnestre cherche d'ailleurs à se décharger de sa responsabilité en accusant le démon qui l'a poussé à agir et qui est l'héritier des fautes antérieures. La notion est néanmoins beaucoup moins présente dans les Choéphores et dans les Euménides. La faute ancestrale est présentée dans Iphigénie en Tauride comme une menace que l'on peut maitriser. Quant à l'Oreste d'Euripide, la notion de faute ancestrale y est régulièrement sollicitée pour se référer au passé de la famille.

10 La faute ancestrale n'est pas présente dans l'œedipe Roi de Sophocle, mais est développée dans les interprétations qui ont été faites de la pièce. Les Sept contre Thèbes, en revanche, imposent la faute ancestrale comme élément central de l'histoire des Labdacides dans les autres traitements qui en ont été faits au cours du ves. L'Antigone 
de Sophocle utilise la faute ancestrale davantage pour caractériser le personnage de la protagoniste, sans en faire un facteur explicatif en soi. Les Phéniciennes d'Euripide en font un usage plus profond, puisque, pour la première fois, les déboires des Labdacides sont expliqués dans la pièce par le meurtre du dragon par Cadmos, causant la colère d'Arès envers la famille pour six générations. L'aboutissement de l'utilisation de la notion apparait dans $\Subset$ dipe à Colone, dernière pièce à appliquer la notion à l'histoire des Labdacides, qui formule de manière précise l'opposition entre volonté individuelle et faute ancestrale.

11 Cet ouvrage surprend son lecteur - dans le bon sens du terme - à plusieurs niveaux. Il va bien plus loin que ce que le titre laisse entendre et ne se limite pas à une simple étude de la punition divine. Au final, c'est un livre dense que nous présente R.G., qui fait preuve d'un haut degré d'érudition. D'un point de vue pratique, on regrettera qu'il ne soit pas toujours aisé de suivre l'analyse minutieuse d'un texte lorsque celui-ci n'est pas reproduit pour le lecteur. La grande force méthodologique de cet ouvrage est de ne pas analyser la faute ancestrale d'un point de vue strictement théologique, mais de toujours remettre en contexte l'utilisation de la notion, afin d'illustrer pourquoi un auteur l'utilise pour faire passer un message à son public. En d'autres termes, c'est l'« agency » de la notion qui est étudiée plus que sa définition.

\section{AUTEURS}

\section{AURIAN DELLI PIZZI}

F.R.S.-FNRS - Université de Liège 